\title{
Correlation between immune-related adverse events and therapeutic effects of nivolumab in patients with malignant pleural mesothelioma
}

Hiroto Yoneda, Hiroshi Nokihara, Atsushi Mitsuhashi, Ryohiko Ozaki, Yohei Yabuki, Hirokazu Ogino, Kenji Otsuka and Yasuhiko Nishioka*

\begin{abstract}
Background: Nivolumab is used for the treatment of malignant pleural mesothelioma (MPM). However, immunerelated adverse events (irAEs) occur in patients treated with nivolumab. Several studies have reported the correlation between irAEs and therapeutic effects of immune checkpoint inhibitor, but none have reported the correlation in MPM. Here we report a retrospective study which shows the correlation between irAEs and therapeutic effects of nivolumab in patients with MPM.
\end{abstract}

Methods: This study included patients treated with nivolumab at Tokushima University Hospital from February 2009 to September 2021. We retrospectively reviewed the medical records to evaluate the several clinical factors, such as the presence or absence of irAEs, their severities, progression-free survival (PFS), overall survival (OS) or objective response to the treatment.

Results: Eleven patients received treatment with nivolumab. Objective response rate was $18.2 \%$ and the disease control rate was $90.9 \%$. Median PFS was 6.8 months (95\% confidence interval, 1.3 to 11.9 months) and median OS was 15.2 months (95\% confidence interval, 8.9 to 21.5 months). IrAEs occurred in eight patients (72.7\%), and grade $\geq 2$ irAEs occurred in six patients (54.5\%). PFS and OS were significantly longer in the grade $\geq 2$ irAEs group than in grade $<2$ irAEs group (median PFS 13.6 vs. 3.8 months, $p=0.0093$; median OS not reached vs. 8.6 months, $p=0.0108$ ).

Conclusions: This is the first study to report the correlation between irAEs and therapeutic effects in patients with MPM. Because the presence of irAEs may be associated with a favorable clinical outcome, early detection and appropriate management of irAEs will increase the therapeutic benefits to patients.

Keywords: Immune-related adverse events, Malignant pleural mesothelioma, Nivolumab, Therapeutic effect

\section{${ }^{*}$ Correspondence: yasuhiko@tokushima-u.ac.jp}

Department of Respiratory Medicine and Rheumatology, Graduate

School of Biomedical Sciences, Tokushima University, 3-18-15,

Kuramoto-cho, Tokushima 770-8503, Japan

\begin{abstract}
Background
Malignant pleural mesothelioma (MPM) is a rare tumor, but it is an aggressive tumor and has a poor prognosis. The median overall survival (OS) is reported to be approximately seven months without any treatments [1], and it is also reported to be 6 to 18 months even with the appropriate treatments, regardless of the therapeutic modalities [2-4]. Many patients with MPM are not
\end{abstract}


offered surgery due to advanced stage, old age, comorbidities, or poor performance status, and are instead considered to be palliative chemotherapy. The combination of cisplatin and pemetrexed is a standard first-line treatment for unresectable MPM [5]. Nivolumab, an anti-programmed cell death protein 1 (PD-1) antibody, has showed an encouraging clinical benefits as a secondor third- line treatment [6,7], and has been approved in Japan since 2018.

The treatment with nivolumab may lead to immunerelated adverse events (irAEs), which sometimes results in the interruption or discontinuation of the treatment [8]. Previous reports in melanoma, non-small-cell lung cancer, and gastric cancer patients have shown that the presence of irAEs with nivolumab were positively associated with their progression-free survival (PFS) and OS [9-13]. However, the correlation between irAEs and outcome of nivolumab for patients with MPM is still unknown. As such, we conducted the retrospective study to investigate whether irAEs are associated with clinical efficacies of nivolumab in MPM.

\section{Methods}

\section{Participants}

Patients, who were diagnosed as MPM in the Department of Respiratory Medicine and Rheumatology at Tokushima University Hospital from February 1st, 2009 to September 30th, 2021, were retrospectively analyzed. Twenty-seven patients were diagnosed with MPM (Fig. 1). Five patients received best supportive care alone, and 22 patients received chemotherapy. 17 of 22 patients received two or more regimens, and eleven patients were treated with nivolumab, and we analyzed these eleven patients in this study. This study was performed in accordance with the Declaration of Helsinki and was approved by the Institutional review board.

\section{Data collection}

We examined several clinical factors including age, gender, Eastern Cooperative Oncology Group (ECOG) performance status (PS), histology, clinical stage, presence or absence of irAEs, the severities of irAEs, PFS, OS and objective response to the treatment. The data were collected retrospectively from the medical records in our hospital.

\section{Treatment and assessment}

Nivolumab was administered intravenously at a dose of $240 \mathrm{mg} /$ body every two weeks. Nivolumab was administered until disease progression or unacceptable adverse events. Adverse events were assessed according to the National Cancer Institute-Common Toxicity Criteria for Adverse Events (NCI-CTCAE) version 5.0. Clinical responses to the treatment were categorized as either complete response (CR), partial response (PR), stable disease (SD), or progressive disease (PD) according to the modified Response Evaluation Criteria in Solid Tumors (mRECIST) or the Response Evaluation Criteria in Solid Tumors (RECIST) version 1.1. The target lesions in pleura were measured uni-dimensionally as the largest tumor thickness perpendicular to the chest wall or mediastinum according to mRECIST [14]; those in nonpleura were measured according to RECIST version 1.1. CR was defined as the disappearance of all target lesions with no evidence of tumor elsewhere, and PR was defined as at least a $30 \%$ reduction in the total tumor measurement. A

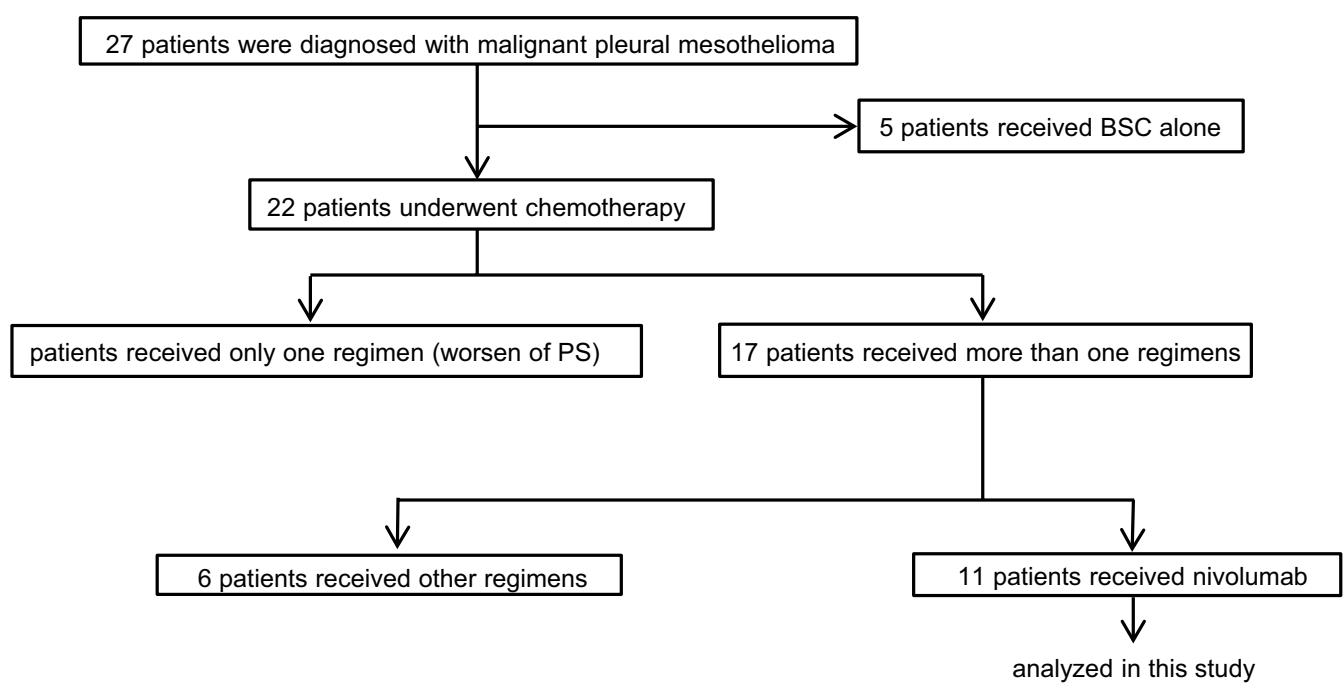

Fig. 1 Flow diagram for study participants. BSC best supportive care, PS performance status, PD progressive disease 
confirmed response required a repeat observation on two occasions 4 weeks apart. PD was defined as an increase of at least $20 \%$ in the total tumor measurement over the nadir measurement, or the appearance of one or more new lesions. Patients with SD were those who fulfilled the criteria for neither PR nor PD. Objective response rate (ORR) was defined as the proportion of $\mathrm{CR}$ and $\mathrm{PR}$, and disease control rate (DCR) was defined as that of CR, PR and SD. PFS was defined as the period from the start of treatment with nivolumab to the date of disease progression. OS was defined as the period from the start of treatment with nivolumab to death or loss of follow-up.

\section{Statistical analysis}

PFS and OS were estimated by the Kaplan-Meier method, and their statistical differences were analyzed by the Logrank test. The statistical analyses were performed using GraphPad Prism version 7 (GraphPad Software, La Jolla, California USA). In this analysis, a $p$ value of $<0.05$ was considered to indicate a significant difference.

\section{Results}

\section{Patient characteristics}

Patient characteristics are shown in Table 1. Among the total eleven patients, eight patients $(72.7 \%)$ were male and three patients $(27.3 \%)$ were female, and median age was 72.0 (56-84) years. Six of eleven patients (54.5\%) have asbestos inhalation history. All patients were histologically epithelial type. All patients received a combination chemotherapy of platinum (five received cisplatin and six received carboplatin) and pemetrexed as a firstline treatment. Ten patients received nivolumab as a second-line and one received as a third-line treatment.

\section{Effects of nivolumab in previously treated MPM patients}

Regarding the best overall response, PR was $18.2 \%$, SD was $72.7 \%$, and PD was $9.1 \%$. The ORR was $18.2 \%$ and the DCR was $90.9 \%$ (Table 2). Median follow-up time was 15.2 months. Median PFS was 6.8 months (95\% confidence interval [CI], 1.3 to 11.9 months) and median OS was 15.2 months (95\% CI, 8.9 to 21.5 months) (Fig. 2).

\section{Onset of irAEs}

The irAEs were observed in total eight patients (72.7\%), and grade $\geq 2$ irAEs were found in six patients (54.5\%) (Tables 3, 4). Two patients were diagnosed with pneumonitis of irAEs by their CT imaging patterns; organizing pneumonia pattern in case 1 and nonspecific interstitial pneumonia pattern in case 3 . The diagnosis was further confirmed by the finding of transbronchial lung biopsy in case 1 , and the clinical finding showing ineffectiveness of broad-spectrum antibiotics in case 3 . In addition, case 2 came to the hospital with disturbed consciousness, and
Table 1 Patient characteristics

\begin{tabular}{|c|c|c|}
\hline No. of patients & 11 & \\
\hline \multicolumn{3}{|l|}{ Gender (\%) } \\
\hline Male & 8 & $(72.7)$ \\
\hline Female & 3 & $(27.3)$ \\
\hline \multicolumn{3}{|l|}{ Age (years) } \\
\hline Median (range) & 72 & $(56-84)$ \\
\hline \multicolumn{3}{|c|}{ ECOG performance status (\%) } \\
\hline 0 & 4 & $(36.4)$ \\
\hline 1 & 6 & $(54.5)$ \\
\hline 2 & 1 & $(9.1)$ \\
\hline \multicolumn{3}{|l|}{ Smoking status (\%) } \\
\hline Never & 4 & $(36.4)$ \\
\hline Ex/current & 7 & $(63.6)$ \\
\hline \multicolumn{3}{|c|}{ Asbestos inhalation history (\%) } \\
\hline+ & 6 & $(54.5)$ \\
\hline- & 5 & $(45.5)$ \\
\hline \multicolumn{3}{|l|}{ Histology (\%) } \\
\hline Epithelial type & 11 & $(100.0)$ \\
\hline \multicolumn{3}{|l|}{ 1st line regimen } \\
\hline CDDP + PEM & 5 & $(45.5)$ \\
\hline$C B D C A+P E M$ & 6 & $(54.5)$ \\
\hline \multicolumn{3}{|l|}{ Regimen line (\%) } \\
\hline 2nd & 10 & $(90.9)$ \\
\hline $3 r d$ & 1 & $(9.1)$ \\
\hline
\end{tabular}

CDDP cisplatin, PEM pemetrexed, CBDCA carboplatin

Table 2 Anti-tumor effect of nivolumab in previously treated malignant pleural mesothelioma patients

\begin{tabular}{lll}
\hline No. of patients & $\mathbf{1 1}$ & \\
\hline Best overall response (\%) & & \\
CR & 0 & $(0.0)$ \\
PR & 2 & $(18.2)$ \\
SD & 8 & $(72.7)$ \\
PD & 1 & $(9.1)$ \\
ORR & $18.2 \%$ & \\
DCR & $90.9 \%$ &
\end{tabular}

$C R$ complete response, $P R$ partial response, $S D$ stable disease, $P D$ progressive disease, $O R R$ objective response rate, $D C R$ disease control rate

showed decreased levels of adrenocorticotropic and cortisol hormones. Magnetic resonance imaging revealed enlargement of the anterior pituitary gland and pituitary stalk, which led to the diagnosis of hypophysitis. The two patients with grade 2 or 3 pneumonitis needed to be treated with corticosteroids and a patient with grade 3 hypophysitis received the corticosteroids replacement therapy. The symptoms of case 1,2 and 3 have been improved by the treatments, but treatments with 

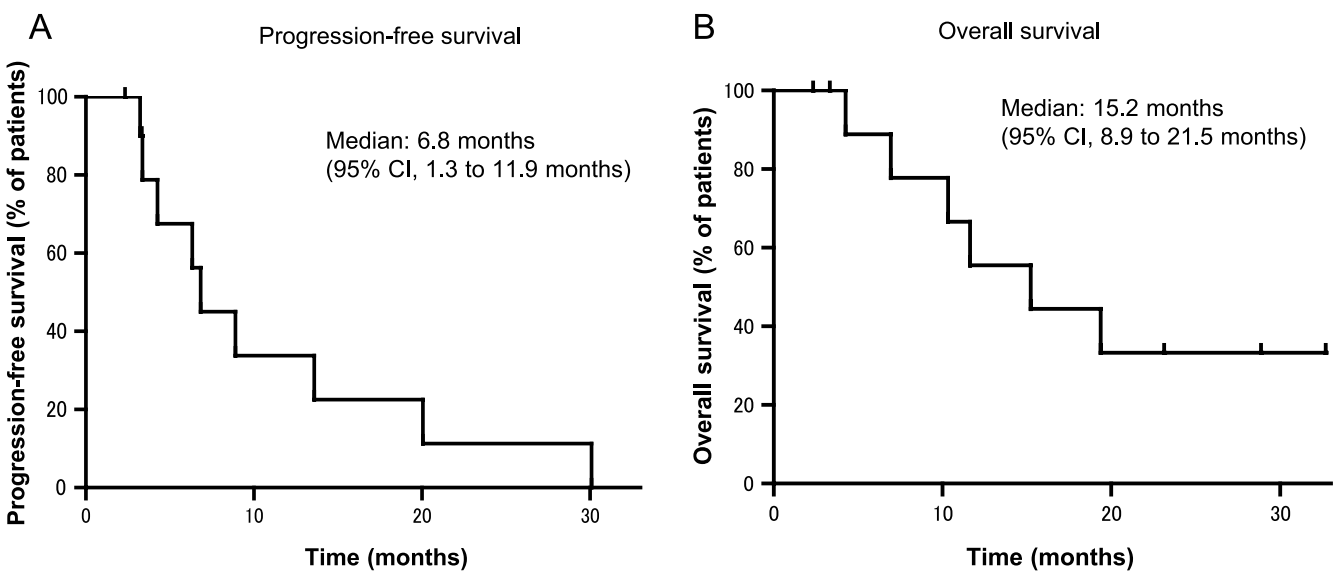

Fig. 2 Kaplan-Meier survival curve of progression free survival (A) and overall survival (B) in previously treated MPM patients

Table 3 Immune-related adverse events (irAEs)

\begin{tabular}{llrll}
\hline & Total & \% & Grade 1 & Grade 2 \\
\hline No. of irAE patients & 8 & 72.7 & & \\
IrAE event (total) & 9 & & 2 & 7 \\
$\quad$ Infusion reaction & 1 & 9.1 & 1 & 0 \\
Itching & 1 & 9.1 & 1 & 0 \\
Hypothyroidism & 2 & 18.2 & 0 & 2 \\
Pneumonitis & 2 & 18.2 & 0 & 2 \\
Hypophysitis & 1 & 9.1 & 0 & 1 \\
Rash maculopapular & 1 & 9.1 & 0 & 1 \\
Bullous dermatitis & 1 & 9.1 & 0 & 1
\end{tabular}

Table 4 Patient details of grade $\geq 2$ irAEs and treatment cycles, OR at the time of irAEs onset

\begin{tabular}{lllll}
\hline Case & irAEs & $\begin{array}{l}\text { Treatment } \\
\text { cycles }\end{array}$ & OR & Continuity \\
\hline 1 & $\begin{array}{l}\text { Grade 3 pneumonitis, grade 2 } \\
\text { hypothyroidism }\end{array}$ & 14 & SD & Discontinue \\
2 & Grade 3 hypophysitis & 18 & SD & Discontinue \\
3 & Grade 2 pneumonitis & 31 & PR & Discontinue \\
4 & Grade 2 hypothyroidism & 3 & SD & Continue \\
5 & Grade 2 rash maculopapular & 17 & SD & Continue \\
6 & Grade 2 bullous dermatitis & 3 & PR & Continue \\
\hline
\end{tabular}

$O R$ objective response, $C R$ complete response, $P R$ partial response, $S D$ stable disease

nivolumab were discontinued. No irAEs-related death was observed in this cohort.

\section{Correlation between irAEs and therapeutic effects}

We next examined the correlation between irAEs and therapeutic effects in patients with MPM treated with nivolumab. We analyzed the therapeutic effects separately for the patients with grade $<2$ irAEs or without irAEs $(n=5)$ and the patients with grade $\geq 2$ irAEs $(n=6)$. Median PFS was significantly longer in the grade $\geq 2$ irAEs group (13.6 months) than in the grade $<2$ irAEs group (3.8 months; $p=0.0093$; Fig. $3 \mathrm{~A}$ ). OS was significantly longer in the grade $\geq 2$ irAEs group than in the grade $<2$ irAEs group ( $p=0.0108$; Fig. 3B).

\section{Discussion}

Nivolumab has been shown to be effective as a second-line treatment of MPM, and the ORR, PFS, and OS were reported to be 24-29\%, 2.6-6.1 months, and 17.3 months, respectively [6, 7]. The treatment effects in the current study were similar to previous reports. In terms of the adverse events, there is a report showing that $32.4 \%$ of patients treated with nivolumab in the second line had treatment-related adverse events of grade 3 or higher [7]. Our study also showed that treatment-related adverse events of grade 3 or higher were observed in two patients $(25.0 \%)$, which was less frequent than the previously report, though the number of patients was small.

Programmed death-ligand 1 (PD-L1) expression in lung cancer $[15,16]$ and microsatellite instability-high (MSI-H) in colorectal cancer [17] are used to predict the therapeutic effects of immune checkpoint inhibitors (ICIs). Among MPM patients, the PD-L1 expression was reported to be negative, positive, and highly positive in 58.2, 41.8, and $9.6 \%$ of the patients, and the high PD-L1 expression was associated with worse OS [18]. In another report, two patients were identified as MSI-H among 83 MPM patients [19], however, the correlation between MSI-H and the therapeutic effects of ICIs in MPM is still unclear. Matsuoka et al. recently analyzed a cohort with various types 

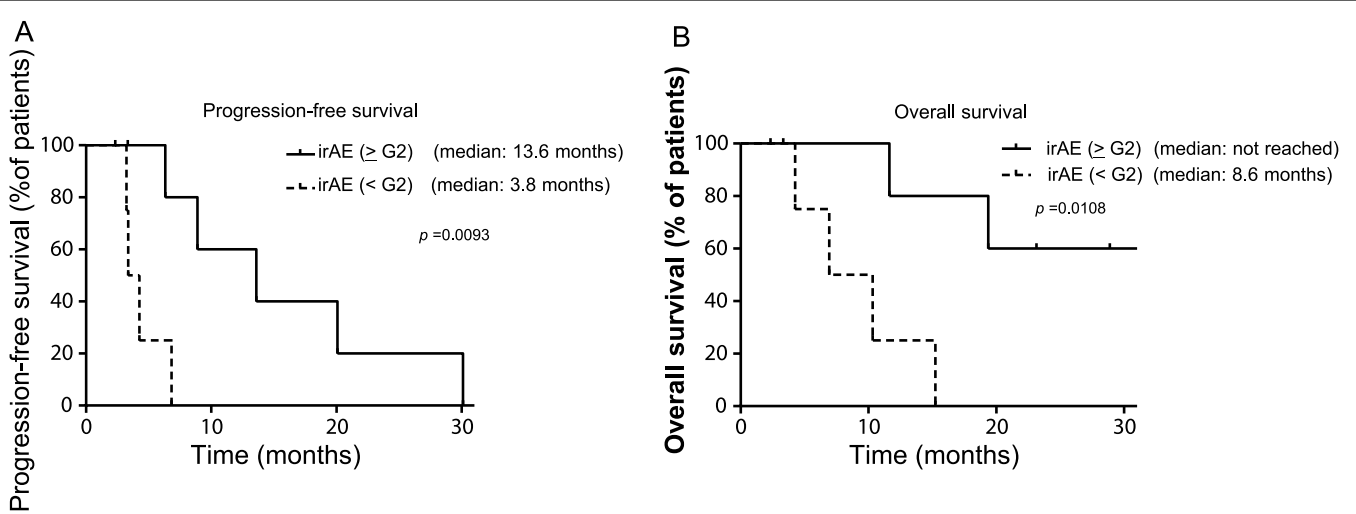

Fig. 3 Correlation between irAEs and therapeutic effects. Kaplan-Meier survival curve of PFS (A) and OS (B). PFS and OS following nivolumab treatment in the grade $\geq 2$ irAEs group $(n=6)$ and grade $<2$ irAEs group $(n=5)$. The median PFS was significantly longer in the grade $\geq 2$ irAEs group than in the grade $<2$ irAEs group ( $p=0.0093$ ). OS was significantly longer in the grade $\geq 2$ irAEs group than in the grade $<2$ irAEs group $(p=0.0108)$

of cancer including MPM, and showed that ORR, OS and PFS were significantly better in the patients with irAEs than in those without irAEs [20]. Although this cohort included only four MPM patients in total 260 cases, none of them developed irAEs, therefore, it was still unclear whether there is a correlation between irAEs and therapeutic effect in patients with MPM. In this study, we examined the eight patients with MPM, and showed the PFS and OS in the patients with irAEs was significantly longer than their counterpart. To our best knowledge, it is a first report identifying the clinical factor which correlates the clinical benefits of ICIs treatment in patients with MPM.

In a phase II study (MERIT study), the treatments of ICIs in 4 out of 34 patients (12\%) had to be terminated due to the adverse events [7]. In the present study, the treatments of nivolumab in two patients with pneumonitis and one patient with hypophysitis were discontinued and not rechallenged. On the other hand, there is another report that rechallenge of ICI was found to be effective in $7.4 \%$ of patients with various types of cancer, and $28.8 \%$ of these patients developed the same irAEs that occurred in the first treatment [21]. These results also support our findings that the ICIs have a potential to show the favorable clinical response in the patients with irAEs, therefore, to prevent the treatment from discontinuing or rechallenge it by careful monitoring and appropriate intervention would improve the outcome in these patients.

Our study has several limitations. Because MPM is a rare disease and nivolumab was approved for patients with MPM in 2018, sample size is small. So we should carefully make a conclusion. It was a retrospective study and conducted in a single institution, so it may contain the selection bias. All histological types were epithelial type, so the correlation between irAEs and therapeutic effects in the other histological types remains unclear.

\section{Conclusions}

This is the first study to report the correlation between irAEs and therapeutic effect of nivolmab in patients with MPM. Because the presence of irAEs may be associated with a favorable PFS and OS, early detection and appropriate management of irAEs would enable us to treat these patients without discontinuation, resulted in improving the therapeutic benefits of this treatment.

\begin{abstract}
Abbreviations
MPM: Malignant pleural mesothelioma; irAEs: Immune-related adverse events; PFS: Progression-free survival; OS: Overall survival; PD-1: Programmed cell death protein 1; ECOG: Eastern cooperative oncology group; PS: Performance status; NCl-CTCAE: National Cancer Institute-Common Toxicity Criteria for Adverse Events; CR: Complete response; PR: Partial response; SD: Stable disease; PD: Progressive disease; mRECIST: Modified response evaluation criteria in solid tumors; RECIST: Response evaluation criteria in solid tumors; ORR: Objective response rate; DCR: Disease control rate; PD-L1: Programmed death-ligand 1; MSI-H: Microsatellite instability-high; ICIs: Immune checkpoint inhibitors.
\end{abstract}

\section{Acknowledgements}

The authors thank the staff of the Department of Respiratory Medicine and Rheumatology of Tokushima University for their advice and assistance.

\section{Authors' contributions}

HY: study design, data collection and analysis and interpretation, paper writing; $\mathrm{HN}$ and $\mathrm{AM}$ : study design, paper editing; $\mathrm{RO}, \mathrm{YY}, \mathrm{HO}$ and $\mathrm{KO}$ : data collection, paper editing; $\mathrm{YN}$ : study design and supervision. All authors read and approved the final manuscript.

\section{Funding}

The author declares no funding support for this study. 


\section{Availability of data and materials}

The datasets used and analyzed during the current study are available from the corresponding author on reasonable request.

\section{Declarations}

Ethics approval and consent to participate

This single-center study was approved by Ethics Committee of Tokushima University. Because of its retrospective nature, the need for written informed consent was waived by the Ethics Committee of Tokushima University. All methods were carried out in accordance with Declaration of Helsinki.

\section{Consent for publication}

Not applicable.

\section{Competing interests}

Department of Respiratory Medicine and Rheumatology of Tokushima University has received research grant funding from Ono Pharmaceutical. HY, $\mathrm{HN}$ and $\mathrm{KO}$ have received speaker fees as honoraria from Ono Pharmaceutical. YN has received speaker fees as honoraria and Scholarship donation from Ono Pharmaceutical. The remaining authors declare that they have no conflicts of interest relevant to the subject of this manuscript.

Received: 13 September 2021 Accepted: 11 November 2021

Published online: 15 November 2021

\section{References}

1. Merritt N, Blewett CJ, Miller JD, Bennett WF, Young JE, Urschel JD. Survival after conservative (palliative) management of pleural malignant mesothelioma. J Surg Oncol. 2001;78:171-4.

2. Ong ST, Vogelzang NJ. Chemotherapy in malignant pleural mesothelioma. A review. J Clin Oncol. 1996;14:1007-17.

3. Aisner J. Current approach to malignant mesothelioma of the pleura. Chest. 1995;107:332S-S344.

4. Carbone M, Adusumilli PS, Alexander HR Jr, Baas P, Bardelli F, Bononi A, et al. Mesothelioma: scientific clues for prevention, diagnosis, and therapy. CA Cancer J Clin. 2019;69:402-29.

5. Vogelzang NJ, Rusthoven JJ, Symanowski J, Denham C, Kaukel E, Ruffie P, et al. Phase III study of pemetrexed in combination with cisplatin versus cisplatin alone in patients with malignant pleural mesothelioma. J Clin Oncol. 2003;21:2636-44.

6. Quispel-Janssen J, van der Noort V, de Vries JF, Zimmerman M, Lalezari F, Thunnissen E, et al. Programmed death 1 blockade with nivolumab in patients with recurrent malignant pleural mesothelioma. J Thorac Oncol. 2018;13:1569-76.

7. Okada M, Kijima T, Aoe K, Kato T, Fujimoto N, Nakagawa K, et al. Clinical efficacy and safety of nivolumab: results of a multicenter, open-label, single-arm, Japanese phase II study in malignant pleural mesothelioma (MERIT). Clin Cancer Res. 2019;25:5485-92.
8. Friedman CF, Proverbs-Singh TA, Postow MA. Treatment of the immunerelated adverse effects of immune checkpoint inhibitors: a review. JAMA Oncol. 2016:2:1346-53.

9. Haratani K, Hayashi H, Chiba Y, Kudo K, Yonesaka K, Kato R, et al. Association of immune-related adverse events with nivolumab efficacy in nonsmall-cell lung cancer. JAMA Oncol. 2018;4:374-8.

10. Nakamura $Y$, Tanaka $R$, Asami $Y$, Teramoto $Y$, Imamura $T$, Sato $S$, et al. Correlation between vitiligo occurrence and clinical benefit in advanced melanoma patients treated with nivolumab: a multi-institutional retrospective study. J Dermatol. 2016;44:117-22.

11. Freeman-Keller M, Kim Y, Cronin H, Richards A, Gibney G, Weber JS. Nivolumab in resected and unresectable metastatic melanoma: characteristics of immune-related adverse events and association with outcomes. Clin Cancer Res. 2016:22:886-94.

12. Teulings HE, Limpens J, Jansen SN, Zwinderman AH, Reitsma JB, Spuls PI, et al. Vitiligo-like depigmentation in patients with stage III-IV melanoma receiving immunotherapy and its association with survival: a systematic review and meta-analysis. J Clin Oncol. 2015;33:773-81.

13. Ando T, Ueda A, Ogawa K, Motoo I, Kajiura S, Nakajima T, et al. Prognosis of immune-related adverse events in patients with advanced gastric cancer treated with nivolumab or pembrolizumab: a multicenter retrospective analysis. In Vivo. 2021;35:475-82.

14. Byrne MJ, Nowak AK. Modified RECIST criteria for assessment of response in malignant pleural mesothelioma. Ann Oncol. 2004;15:257-60.

15. Garon EB, Rizvi NA, Hui R, Leighl N, Balmanoukian AS, Eder JP, et al. Pembrolizumab for the treatment of non-small-cell lung cancer. N Engl J Med. 2015:372:2018-28.

16. Herbst RS, Giaccone G, de Marinis F, Reinmuth N, Vergnenegre A, Barrios $\mathrm{CH}$, et al. Atezolizumab for first-line treatment of PD-L1-selected patients with NSCLC. N Engl J Med. 2020;383:1328-39.

17. Le DT, Kim TW, Van Cutsem E, Geva R, Jäger D, Hara H, et al. Phase II openlabel study of pembrolizumab in treatment-refractory, microsatellite instability-high/mismatch repair-deficient metastatic colorectal cancer: KEYNOTE-164. J Clin Oncol. 2020;38:11-9.

18. Thapa B, Salcedo A, Lin X, Walkiewicz M, Murone C, Ameratunga M, et al. The immune microenvironment, genome-wide copy number aberrations, and survival in mesothelioma. J Thorac Oncol. 2017;12:850-9.

19. Bonneville R, Krook MA, Kautto EA, Miya J, Wing MR, Chen HZ, et al. Landscape of microsatellite instability across 39 cancer types. JCO Precis Oncol. 2017. https://doi.org/10.1200/PO.17.00073.

20. Matsuoka H, Hayashi T, Takigami K, Imaizumi K, Shiroki R, Ohmiya N, et al. Correlation between immune-related adverse events and prognosis in patients with various cancers treated with anti PD-1 antibody. BMC Cancer. 2020;20:656.

21. Dolladille C, Ederhy S, Sassier M, Cautela J, Thuny F, Cohen AA, et al. Immune checkpoint inhibitor rechallenge after immune-related adverse events in patients with cancer. JAMA Oncol. 2020;6:865-71.

\section{Publisher's Note}

Springer Nature remains neutral with regard to jurisdictional claims in published maps and institutional affiliations.
Ready to submit your research? Choose BMC and benefit from:

- fast, convenient online submission

- thorough peer review by experienced researchers in your field

- rapid publication on acceptance

- support for research data, including large and complex data types

- gold Open Access which fosters wider collaboration and increased citations

- maximum visibility for your research: over $100 \mathrm{M}$ website views per year

At BMC, research is always in progress.

Learn more biomedcentral.com/submissions 